\title{
Infrared absorption of the charge-ordering phase: Lattice effects
}

\author{
C.A. Perroni, V. Cataudella, G. De Filippis, G. Iadonisi, V. Marigliano Ramaglia, and F. \\ Ventriglia \\ Coherentia-INFM and Dipartimento di Scienze Fisiche, \\ Università degli Studi di Napoli "Federico II", \\ Complesso Universitario Monte Sant'Angelo, \\ Via Cintia, I-80126 Napoli, Italy
}

(November 10, 2018)

The phase diagram of the half-filled spinless Holstein model for electrons interacting with quantum phonons is derived in three dimensions extending at finite temperature $T$ a variational approach introduced for the one-dimensional $T=0$ case. Employing the variational scheme, the spectral and optical properties of the system are evaluated in the different regimes that characterize the normal and ordered state. The effects of the chargeordering $(C O)$ induce a transfer of spectral weight from low to high energies in the conductivity spectra, as the temperature decreases or the strength of the electron-phonon $(e l-p h)$ interaction increases. The inclusion of effects of lattice fluctuations is able to smooth the inverse square-root singularity expected for the case of the mean-field approach and determines a subgap tail absorption. Moreover, in the weak to intermediate coupling regime, a twocomponent structure is obtained within the $C O$ phase at low frequency: the remnant Drude-like term and the incipient absorption band centered around the gap energy. 
In the last years there has been a renewed interest in charge density wave $(C D W)$ materials. ${ }^{1}$ The transition to a $C O$ phase is common to a wide range of compounds, ${ }^{2}$ including quasi-one-dimensional organic conductors, ${ }^{1,3}$ dichalcogenides, ${ }^{4,5}$ molybdenum bronzes ${ }^{2,6}$ and A-15 materials. ${ }^{7}$ Moreover, the $C O$ transition has been associated with the stripe density wave order in some cuprates ${ }^{8}$ and nickelates,,${ }^{9,10}$ with the commensurate or incommensurate charge ordering in manganites. ${ }^{11}$

The ordered phase generally evolves out of a metallic phase. In the weak $e l-p h$ coupling regime the transition is the well-understood $C D W$ instability of the Fermi liquid. ${ }^{1}$ The related equations predict a ratio of $T=0$ gap $\Delta$ to the ordering temperature $T_{C O}$ close to the $B C S$ value $\Delta / k_{B} T_{C O}=3.52$. However in quasi-one-dimensional materials this ratio assumes larger values because of critical fluctuations in low dimensionality. Furthermore values of the ratio larger than 10 have been measured in quasi-two-dimensional and threedimensional materials. ${ }^{4,5,7,12-14}$ In $N i$ compounds $^{12} \Delta / k_{B} T_{C O} \simeq 13$ and in manganites ${ }^{14}$ a ratio of order of 30 have been deduced by the energy gap fitted by optical measurements. This suggests that a strong coupling $C O$ phase develops in novel materials and that the lattice degrees of freedom can be important for stabilizing the ordered state. ${ }^{15} \mathrm{~A}$ very useful approach to investigate the properties of the ordered state is to study the optical absorption of $C O$ materials. ${ }^{1,15-18}$ In particular the lattice fluctuation effects ${ }^{19-21}$ and the response of the system in the strong coupling $C O$ regime ${ }^{12,15,22}$ have been studied. Indeed the inverse square-root singularity expected for the case of a static distorted lattice can be removed by the fluctuation effects giving rise to a subgap tail absorption. ${ }^{20}$ Furthermore the presence of a polaronic peak in the $M I R$ band of $N i$ compounds observed above and below the transition temperature can be explained only within a theory valid for intermediate to strong coupling regime. ${ }^{12,15}$

Theoretically one of the simplest models of electrons interacting with phonons and developing a $C O$ state is the Holstein model. ${ }^{23}$ The $C D W$ correlations and the transition temperature have been studied within this model by Monte Carlo simulations ${ }^{24-28}$ showing that the ordered phase is stable at half-filling. Recently, using the dynamical mean-field 
theory $(D M F T)$, the issue of the non-perturbative coupling scenario of the $C O$ phase and of the origin of the large ratio of the zero temperature gap to the transition temperature has been addressed. ${ }^{29-31}$ The results point out that the scattering of electrons by the phonons involved in the lattice distortions represents the crucial effect in the coupling regime relevant to most $C D W$ materials.

In this paper we extend the variational scheme proposed for the spinless Holstein model at half-filling by H. Zheng, D. Feinberg and M. Avignon ${ }^{32}(Z F A)$ to finite temperature. We note that at zero temperature the $Z F A$ results are in good agreement with different numerical works. ${ }^{33-35}$ Actually this approach introduces lattice fluctuations on the Peierls dimerization since it takes into account the nonadiabatic polaron formation. Within this scheme all electrons in the Fermi sea are involved in the $e l-p h$ scattering leading, above the $C O$ transition, to a stable phase of disordered small polarons $(S P)$ in the intermediate to strong coupling regime. Thus, at half-filling the phase diagram is consistent with previous DMFT results pointing out a strong dependence of the transition temperature on the adiabaticity particularly in the weak to intermediate coupling regime. ${ }^{29-31}$ Within the $Z F A$ scheme we have derived the spectral properties useful to characterize the different phases of the system. Next, by using the formalism of generalized Matsubara Green's functions ${ }^{36-40}$, we have determined the scattering rate of the quasi-particles finding that, in the $C O$ phase, the single-phonon emission and absorption represent the main mechanism of damping. The lattice fluctuation effects included in the scattering rate are beyond the $Z F A$ scheme and modify the density of states that, this way, is able to capture the features of the intermediate coupling regime in agreement with previous DMFT studies. ${ }^{29-31}$

The scattering rate turns out fundamental also to derive the optical properties of the system. With decreasing $T$ or increasing the $e l-p h$ coupling, our conductivity spectra are characterized by a transfer of spectral weight from low to high energies with the opening of an optical gap. The effect of the lattice fluctuations is able to smooth the inverse square-root singularity of the mean-field approach in the optical absorption and induces a subgap tail. In the weak to intermediate coupling regime, a two-peak structure is obtained within the 
$C O$ phase at low frequency: the remnant Drude-like term and the incipient absorption band centered around the gap energy.

In section I the model and the variational approach are reviewed; in section II the spectral properties are discussed; in section III the damping of the particle motion is calculated; in section IV the optical properties are deduced.

\section{MODEL AND VARIATIONAL APPROACH}

In this paper we study the spinless Holstein model at half-filling. ${ }^{23}$ The Hamiltonian reads

$$
\begin{aligned}
H= & -t \sum_{<i, j>} c_{i}^{\dagger} c_{j}+\omega_{0} \sum_{i} a_{i}^{\dagger} a_{i}+g \omega_{0} \sum_{i} c_{i}^{\dagger} c_{i}\left(a_{i}+a_{i}^{\dagger}\right) \\
& -\mu \sum_{i} c_{i}^{\dagger} c_{i} .
\end{aligned}
$$

Here $t$ is the electron transfer integral between nearest neighbor $(n n)$ sites $\langle i, j\rangle, c_{i}^{\dagger}\left(c_{i}\right)$ creates (destroys) an electron at the i-th site and $\mu$ is the chemical potential. In the second term of eq.(1) $a_{i}^{\dagger}\left(a_{i}\right)$ is the creation (annihilation) phonon operator at the site i, $\omega_{0}=\sqrt{k / M}$ denotes the frequency of the optical phonon mode, with $k$ restoring force per length unit and $M$ mass of the local oscillator. The parameter $g$ represents the coupling constant between electrons and phonons

$$
g=\frac{A}{\omega_{0} \sqrt{2 M \omega_{0}}}
$$

where $A$ is the energy per displacement unit due to the coupling of the charge carriers with the lattice. The dimensionless parameter $\lambda$

$$
\lambda=\frac{g^{2} \omega_{0}}{2 D t}
$$

with $D$ dimensionality of the system, indicating the ratio between the $S P$ binding energy and the energy gain of an itinerant electron on a rigid lattice, is useful to measure the strength of the $e l-p h$ interaction in the adiabatic regime. We consider spinless electrons 
since they, even if at a very rough level, mimic the action of an on-site Coulomb repulsion preventing the formation of local pairs.

The hopping of electrons is supposed to take place between the equivalent $n n$ sites of a simple cubic lattice separated by the distance $\left|n-n^{\prime}\right|=a$. The units are such that the Planck constant $\hbar=1$, the Boltzmann constant $k_{B}=1$ and the lattice parameter $a=1$.

Following the ZFA variational scheme, ${ }^{32}$ we perform three successive canonical transformations to treat the electron-phonon interaction variationally and to introduce the chargeordering solution. The first is the variational Lang-Firsov unitary transformation ${ }^{32}$

$$
U_{1}=\exp \left[g \sum_{j}\left(f c_{j}^{\dagger} c_{j}+\Delta_{j}\right)\left(a_{j}-a_{j}^{\dagger}\right)\right],
$$

where $f$ and $\Delta_{j}$ are variational parameters. The quantity $f$ controls the degree of the polaron effect and $\Delta_{i}$ denotes a displacement field describing lattice distortions due to the average electron motion. The second transformation is

$$
U_{2}=\exp \left[\alpha \sum_{j}\left(a_{j}^{\dagger} a_{j}^{\dagger}-a_{j} a_{j}\right)\right]
$$

where the variational parameter $\alpha$ determines a phonon frequency renormalization. The transformed Hamiltonian $\tilde{H}=U_{2}^{-1} U_{1}^{-1} H U_{1} U_{2}$ is

$$
\begin{aligned}
\tilde{H}= & -t \sum_{<i, j>} X_{i}^{\dagger} X_{j} c_{i}^{\dagger} c_{j}+\bar{\omega}_{0} \sum_{i} a_{i}^{\dagger} a_{i}+L \omega_{0} \sinh ^{2}(2 \alpha)+g^{2} \omega_{0} \sum_{i} \Delta_{i}^{2} \\
& +\omega_{0} \sinh (2 \alpha) \cosh (2 \alpha) \sum_{i}\left(a_{i}^{\dagger} a_{i}^{\dagger}+a_{i} a_{i}\right)-g \omega_{0} e^{2 \alpha} \sum_{i} \Delta_{i}\left(a_{i}+a_{i}^{\dagger}\right) \\
& +g \omega_{0}(1-f) e^{2 \alpha} \sum_{i} c_{i}^{\dagger} c_{i}\left(a_{i}+a_{i}^{\dagger}\right)+\sum_{i} c_{i}^{\dagger} c_{i}\left(\eta_{i}-\mu\right),
\end{aligned}
$$

where we have the phonon operator $X_{i}$

$$
X_{i}=\exp \left[g f e^{-2 \alpha}\left(a_{i}-a_{i}^{\dagger}\right)\right]
$$

the renormalized phonon frequency $\bar{\omega}_{0}=\omega_{0} \cosh (4 \alpha)$, the number of lattice sites $L$ and the quantity $\eta_{i}$

$$
\eta_{i}=g^{2} \omega_{0} f(f-2)+2 g^{2} \omega_{0}(f-1) \Delta_{i} .
$$


At half-filling the charge-ordered solution is obtained by assuming

$$
\Delta_{i}=\Delta+\Delta_{C O} e^{i \vec{Q} \cdot \vec{R}_{i}}
$$

where $\Delta$ represents the lattice distortion unaffected by the instantaneous position of electrons and $\Delta_{C O}$ the additional local lattice distortion due to the Peierls dimerization with $\vec{Q}=$ $(\pi, \pi, \pi)$.

In the ZFA approach the free energy is deduced employing the Bogoliubov inequality and introducing a test Hamiltonian characterized by non interacting electron and phonon degrees of freedom such that $\left\langle\tilde{H}-H_{\text {test }}\right\rangle_{t}=0$, where $<>_{t}$ indicates a thermodynamic average obtained by using $H_{\text {test }}$. The test Hamiltonian is given by

$$
\begin{aligned}
H_{\text {test }}= & -t_{\text {eff }} \sum_{<i, j>} c_{i}^{\dagger} c_{j}+\bar{\omega}_{0} \sum_{i} a_{i}^{\dagger} a_{i}+L \omega_{0} \sinh ^{2}(2 \alpha)+L g^{2} \omega_{0}\left(\Delta^{2}+\Delta_{C O}^{2}\right) \\
& -2 g^{2} \omega_{0} \Delta_{C O}(1-f) \sum_{i} e^{i \vec{Q} \cdot \vec{R}_{i}} c_{i}^{\dagger} c_{i}-\mu_{0} \sum_{i} c_{i}^{\dagger} c_{i},
\end{aligned}
$$

where the subsidiary chemical potential $\mu_{0}$ is

$$
\mu_{0}=\mu-g^{2} \omega_{0} f(f-2)+2 g^{2} \omega_{0}(f-1) \Delta .
$$

The quantity $t_{e f f}=t e^{-S_{T}}$ denotes the effective transfer integral, where the quantity

$$
S_{T}=g^{2} f^{2} e^{-4 \alpha}\left(2 N_{0}+1\right)
$$

controls the band renormalization due to the antiadiabatic polaron effect, with $N_{0}$ the average number of phonons with frequency $\bar{\omega}_{0}$. The test Hamiltonian is diagonalized by a third canonical Bogoliubov transformation ${ }^{32}$ yealding

$$
\begin{aligned}
\tilde{H}_{\text {test }}= & \sum_{\mathbf{k} \in N Z}\left(\xi_{\mathbf{k}}^{(+)}-\mu_{0}\right) d_{\mathbf{k}}^{\dagger} d_{\mathbf{k}}+\sum_{\mathbf{k} \in N Z}\left(\xi_{\mathbf{k}}^{(-)}-\mu_{0}\right) p_{\mathbf{k}}^{\dagger} p_{\mathbf{k}}+\bar{\omega}_{0} \sum_{\mathbf{q}} a_{\mathbf{q}}^{\dagger} a_{\mathbf{q}}+ \\
& +L \omega_{0} \sinh ^{2}(2 \alpha)+L g^{2} \omega_{0}\left(\Delta^{2}+\Delta_{C O}^{2}\right)
\end{aligned}
$$

where $d_{\mathbf{k}}^{\dagger}\left(d_{\mathbf{k}}\right)$ creates (destroys) a quasi-particle in the upper band $\xi_{\mathbf{k}}^{(+)}=\sqrt{\tilde{\epsilon}_{\mathbf{k}}^{2}+E^{2}}, p_{\mathbf{k}}^{\dagger}\left(p_{\mathbf{k}}\right)$ creates (destroys) a quasi-particle in the lower band $\xi_{\mathbf{k}}^{(-)}=-\sqrt{\tilde{\epsilon}_{\mathbf{k}}^{2}+E^{2}}$ and $\tilde{\epsilon}_{\mathbf{k}}$ is the polaronic band. We note that $N Z$ indicates the New Brillouin Zone defined by the condition $\tilde{\epsilon}_{\mathbf{k}} \leq 0$. 
In the charge-ordered phase a gap opens between the upper and lower bands in the quasiparticle spectrum and it is twice the quantity

$$
E=2 g^{2} \omega_{0} \Delta_{C O}(1-f)
$$

At half-filling $(\rho=0.5)$, the variational free energy of the system is

$$
\frac{F}{L}=f^{e l}+T \log \left(1-e^{-\beta \bar{\omega}_{0}}\right)+\omega_{0} \sinh ^{2}(2 \alpha)+g^{2} \omega_{0}\left(\Delta^{2}+\Delta_{C O}^{2}\right)+\mu(\rho) \rho
$$

where $\beta$ is the inverse of the temperature. The electron free energy reads

$$
f^{e l}=T \int_{-\tilde{W}}^{0} d \epsilon g(\epsilon) \log \left[n_{F}\left(\xi^{(+)}\right) n_{F}\left(\xi^{(-)}\right)\right]
$$

where $g(\epsilon)$ is the density of states, $\tilde{W}=6 t_{\text {eff }}$ is the renormalized band half-width, $n_{F}(\epsilon)$ is the Fermi distribution, $\xi^{(+)}=\sqrt{\epsilon^{2}+E^{2}}$ and $\xi^{(-)}=-\xi^{(+)}$. In order to simplify the calculations, in the three dimensional case we consider a semicircular density of states

$$
g(\epsilon)=\left(\frac{2}{\pi \tilde{W}^{2}}\right) \theta(\tilde{W}-|\epsilon|) \sqrt{\tilde{W}^{2}-\epsilon^{2}}
$$

where $\theta(x)$ is the Heaviside function. Actually $g(\epsilon)$ represents a simple approximate expression for the exact density of states and it is generally used for a 3-D lattice. ${ }^{42,43}$

Within the variational approach the kinetic energy mean value $<\hat{T}>$

$$
<\hat{T}>=\int_{-\tilde{W}}^{0} d \epsilon g(\epsilon) \frac{\epsilon^{2}}{\sqrt{\epsilon^{2}+E^{2}}}\left[n_{F}\left(\xi^{(+)}\right)-n_{F}\left(\xi^{(-)}\right)\right]
$$

and the electron order parameter $m_{e}$

$$
m_{e}=\left(\frac{1}{L}\right) \sum_{i} e^{i \vec{Q} \cdot \vec{R}_{i}}\left\langle c_{i}^{\dagger} c_{i}\right\rangle=E \int_{-\tilde{W}}^{0} d \epsilon \frac{g(\epsilon)}{\sqrt{\epsilon^{2}+E^{2}}}\left[n_{F}\left(\xi^{(-)}\right)-n_{F}\left(\xi^{(+)}\right)\right]
$$

can be evaluated. The $C O$ phase is characterized by the order parameter different from zero.

In this paper we discuss results valid in three dimensions. In Fig.1 we report the phase diagram obtained within our approach for $W=6 \omega_{0}$, with $W$ the bare band half-width. We compare it with the mean-field result obtained in the adiabatic limit for $f=0$ and $\alpha=0$ 
that overestimates the transition temperature. The $A$ phase represents the Fermi-liquidlike normal state, while the $B$ phase the disordered localized $S P$ normal state. This latter phase is determined when there is absence of order $\left(\Delta_{C O}=0\right)$ and the lattice presents the largest distortions $(f=1)$. While the transition from $A$ to $C O$ is continuous, the transition from $C O$ to $B$ and the crossover from $A$ to $B$ is rather discontinuous. We stress that a discontinuous character for the transition can be considered as a drawback of the $Z F A$ approach. ${ }^{32}$ However, the phase diagram bears a strong resemblance to that derived with more sophisticated tecniques. ${ }^{29,30}$

In the inset of Fig.1, the ratio of $T=0$ gap $\Delta$ to the ordering temperature $T_{C O}$ is shown. It is interesting to note that within the $C O$ phase it is possible to distinguish two regimes. The first is the mean-field regime in the sense that the ratio assumes values near the $B C S \Delta / k_{B} T_{C O}=3.52$, while the second regime exhibits larger values of this quantity. For instance at $\lambda=0.9$ the ratio $\Delta / k_{B} T_{C O}$ is about 13 , the value measured in nickelates. ${ }^{12}$ Hence, a strong coupling $C O$ phase develops starting from intermediate values of the $e l-p h$ coupling. This $C O$ regime is characterized by a gap of the order of $2 g^{2} \omega_{0}$ and by distortions comparable to those of the disordered $S P$ phase.

Previous DMFT results ${ }^{31}$ have pointed out a strong dependence of the transition temperature on the adiabaticity particularly in the weak to intermediate coupling regime. Therefore we have determined the transition temperature for two different values of the adiabaticity ratio (see Fig.2a ). The temperature $T_{C O}$ strongly depends on the adiabaticity for intermediate $e l-p h$ couplings. Here the lattice fluctuations are very effective in reducing $T_{C O}$. The variation $\Delta T_{C O}=T_{C O}^{*}-T_{C O}$ of the transition temperatures due to different masses of the oscillators is reported in Fig. $2 \mathrm{~b}$. The ratio between the old and the new masses simulates the isotopic substitution of the oxygen from ${ }^{16} \mathrm{O}(M)$ to ${ }^{18} \mathrm{O}\left(M^{*}\right)$. Actually in many perovskite oxides the presence of the oxygen atoms induces the $e l-p h$ coupling. ${ }^{44}$ Therefore we can consider a change of the values of $\omega_{0}$ and $g$ to $\omega_{0}^{*}=\omega_{0} \sqrt{M / M^{*}}$ and $g^{*}=g\left(M^{*} / M\right)^{1 / 4}$ respectively. ${ }^{45}$ The softening of the phonon frequency induces an increase of the transition temperature. Furthemore, as a function of $\lambda$, the difference between the two ordering tem- 
peratures is characterized by a maximum in the region of weak to intermediate $e l-p h$ couplings.

\section{SPECTRAL PROPERTIES}

In this section we calculate the spectral properties within the $Z F A$ approach. They are discussed in order to characterize the different phases of the diagram reported in Fig.1.

The electron Matsubara Green's function can be disentangled into electronic and phononic terms ${ }^{46}$ by using the test Hamiltonian (10), hence

$$
\mathcal{G}(\mathbf{k}, \tau)=-\left(\frac{1}{L}\right) \sum_{i, j} e^{i \mathbf{k} \cdot\left(\mathbf{R}_{\mathbf{i}}-\mathbf{R}_{\mathbf{j}}\right)}\left\langle T_{\tau} \bar{c}_{i}(\tau) c_{j}^{\dagger}\right\rangle_{t}\left\langle T_{\tau} \bar{X}_{i}(\tau) X_{j}^{\dagger}\right\rangle_{t},
$$

where we have $\bar{c}_{i}(\tau)=e^{\tau H_{t}} c_{i} e^{-\tau H_{t}}$ and $\bar{X}_{i}(\tau)=e^{\tau H_{t}} X_{i} e^{-\tau H_{t}}$. The Green's function in Matsubara frequencies $\omega_{n}$ becomes

$$
\begin{aligned}
\mathcal{G}\left(\mathbf{k}, i \omega_{n}\right)= & e^{-S_{T}} \mathcal{G}^{(c)}\left(\mathbf{k}, i \omega_{n}\right)+ \\
& e^{-S_{T}}\left(\frac{1}{N}\right) \sum_{\mathbf{k}_{\mathbf{1}}}\left(\frac{1}{\beta}\right) \sum_{m} \mathcal{G}^{(c)}\left(\mathbf{k}_{1}, i \omega_{m}\right) \int_{0}^{\beta} e^{\left(i \omega_{n}-i \omega_{m}\right) \tau}\left\{e^{s \cosh \left[\bar{\omega}_{0}\left(\tau-\frac{\beta}{2}\right)\right]}-1\right\},
\end{aligned}
$$

where $s$ is

$$
s=2 f^{2} g^{2} e^{-4 \alpha}\left[N_{0}\left(N_{0}+1\right)\right] .
$$

In eq. $(21) \mathcal{G}^{(c)}\left(\mathbf{k}, i \omega_{n}\right)$ is the Green's function

$$
\mathcal{G}^{(c)}\left(\mathbf{k}, i \omega_{n}\right)=u_{\mathbf{k}}^{2} \mathcal{G}^{(+)}\left(\mathbf{k}, i \omega_{n}\right)+v_{\mathbf{k}}^{2} \mathcal{G}^{(-)}\left(\mathbf{k}, i \omega_{n}\right)
$$

where $\mathcal{G}^{(+)}\left(\mathbf{k}, i \omega_{n}\right)$ and $\mathcal{G}^{(-)}\left(\mathbf{k}, i \omega_{n}\right)$ are the Green's functions associated to the quasiparticles of the upper and lower bands, with $u_{\mathbf{k}}^{2}$ given by

$$
u_{\mathbf{k}}^{2}=\frac{1}{2}\left[1+\frac{\tilde{\epsilon}_{\mathbf{k}}}{\sqrt{\tilde{\epsilon}_{\mathbf{k}}^{2}+E^{2}}}\right]
$$

and $v_{\mathbf{k}}^{2}$ by

$$
v_{\mathbf{k}}^{2}=\frac{1}{2}\left[1-\frac{\tilde{\epsilon}_{\mathbf{k}}}{\sqrt{\tilde{\epsilon}_{\mathbf{k}}^{2}+E^{2}}}\right] .
$$


Two physically distinct terms ${ }^{46}$ appear in the Eq.(21). The first derives from the coherent motion of quasi-particles and their surrounding phonon cloud, while the incoherent term is due to processes changing the number of phonons during the hopping of the charges.

Making the analytic continuation $i \omega_{n} \rightarrow \omega+i \delta$, one obtains the retarted Green's function $G_{r e t}(\mathbf{k}, \omega)$ and the spectral function

$$
\begin{aligned}
A(\mathbf{k}, \omega)= & -2 \Im G_{r e t}(\mathbf{k}, \omega)=2 \pi e^{-S_{T}}\left[u_{\mathbf{k}}^{2} \delta\left(\omega-\xi_{\mathbf{k}}^{(+)}\right)+v_{\mathbf{k}}^{2} \delta\left(\omega-\xi_{\mathbf{k}}^{(+)}\right)\right]+ \\
& 2 \pi e^{-S_{T}} \sum_{l(\neq 0)-\infty}^{+\infty} I_{l}(s) e^{\frac{\beta l \bar{\omega}_{0}}{2}}\left[1-n_{F}\left(\omega-l \bar{\omega}_{0}\right)\right] K\left(\omega-l \bar{\omega}_{0}\right)+ \\
& 2 \pi e^{-S_{T}} \sum_{l(\neq 0)-\infty}^{+\infty} I_{l}(s) e^{\frac{\beta l \bar{\omega}_{0}}{2}} n_{F}\left(\omega+l \bar{\omega}_{0}\right) K\left(\omega+l \bar{\omega}_{0}\right)+ \\
& 2 \pi e^{-S_{T}}\left[I_{0}(s)-1\right] K(\omega),
\end{aligned}
$$

where the function $K(\omega)$ is

$$
K(\omega)=H(\omega)+H(-\omega),
$$

with

$$
H(\omega)=\frac{g\left(\sqrt{\omega^{2}-E^{2}}\right)}{\sqrt{1-\frac{E^{2}}{\omega^{2}}}} \theta(\omega-E) \theta\left(\sqrt{E^{2}+W^{2}}-\omega\right) .
$$

In the normal phase the first term of eq.(26) represents the purely polaronic band contribution and shows a delta behavior. In the $C O$ phase, this term is equal to the result of the mean-field approach when one neglects the renormalization of the upper and lower band due to the polaron effect. The remaining terms of eq.(26) provide the incoherent contribution that spreads over a wide energy range.

Thus we get the renormalized density of states $N(\omega)$ that is normalized to unity. In Fig.3a we report the density of states for three different values of the $e l-p h$ coupling at $T=0.023 W$. It is apparent that in the normal phase $(\lambda=0.15)$ the weight of the coherent term is prevalent and the density of states is practically unchanged with respect to the noninteracting case. Entering the $C O$ phase, a gap opens in the quasi-particle spectrum and it broadens with increasing the parameter $\lambda$. We note that, at the energies corresponding to 
the gap, the inverse square-root singularity occurs. The other sharp features of the density of states are due to one-phonon and two-phonon processes in the upper and lower bands.

At higher temperatures (Fig. 3b) similar features are found. However, in this case, for large $e l-p h$ couplings, the density of states is determined by the incoherent dynamics of the small polaron excitations. ${ }^{46}$ It is made up of two bands peaked approximatively around $-g^{2} \omega_{0}$ and $g^{2} \omega_{0}$, whose heights are equal at half-filling. We stress the evolution of the gap energy of the $C O$ phase toward the characteristic energy $2 g^{2} \omega_{0}$ that represents the energy range between the two peaks of the $S P$ density of states.

\section{DAMPING}

In this section we deal with the $e l-p h$ self-energy that includes lattice fluctuation effects beyond the $Z F A$ approach. This quantity allows to determine the scattering rate of the quasi-particles of the upper and lower bands and a better density of states. This analysis will play an essential role in the infrared absorption calculations that is the main aim of this paper. For sake of clarity we will put forward this discussion.

Retaining only the dominant autocorrelation terms at the second step of iteration ${ }^{36-40}$, we derive the local self-energy

$$
\Sigma^{(2)}\left(i \omega_{n}\right)=\left(\frac{1}{N}\right) \sum_{\mathbf{k}_{1}}\left(\frac{1}{\beta}\right) \sum_{m} \mathcal{G}^{(c)}\left(\mathbf{k}_{1}, i \omega_{m}\right) \int_{0}^{\beta} d \tau e^{\left(i \omega_{n}-i \omega_{m}\right) \tau}\left[f_{1}(\tau)+f_{2}(\tau)\right],
$$

where $f_{1}(\tau)$ is

$$
f_{1}(\tau)=Z t^{2} e^{-2 S_{T}}\left[e^{z \cosh \left[\bar{\omega}_{0}\left(\tau-\frac{\beta}{2}\right)\right]}-1\right],
$$

$z=2 s$, with $s$ given by eq. (22), $Z$ the number of $n n$ sites, and $f_{2}(\tau)$ is

$$
f_{2}(\tau)=2 g^{2} \omega_{0}^{2} e^{4 \alpha}(1-f)^{2}\left[N_{0}\left(N_{0}+1\right)\right]^{\frac{1}{2}} \cosh \left[\bar{\omega}_{0}\left(\tau-\frac{\beta}{2}\right)\right] .
$$

Other terms beyond the autocorrelation ones induce a dependence of the self-energy on the momentum and could be included. ${ }^{40}$ We note that the fundamental autocorrelation contribution presents some analogies with the corresponding self-energy within DMFT approaches 
where the independence on the momentum is the main assumption when the interaction term is local.

Making the analytic continuation $i \omega_{n} \rightarrow \omega+i \delta$, we can calculate the scattering rate of the quasi-particles of the upper band

$$
\Gamma_{+}(\mathbf{k})=\tilde{\Gamma}\left(\omega=\xi_{\mathbf{k}}^{(+)}\right)=-2 \Im \Sigma_{r e t}^{(2)}\left(\omega=\xi_{\mathbf{k}}^{(+)}\right)
$$

We can express the scattering rate in the following way

$$
\Gamma_{+}(\mathbf{k})=\Gamma_{+}\left(\xi_{\mathbf{k}}^{(+)}\right)=\Gamma_{1-p h o n}\left(\xi_{\mathbf{k}}^{(+)}\right)+\Gamma_{\text {multi-phon }}\left(\xi_{\mathbf{k}}^{(+)}\right)+\Gamma_{r e s}\left(\xi_{\mathbf{k}}^{(+)}\right)
$$

where $\Gamma_{1-p h o n}$ is the contribution due to single phonon processes only

$$
\begin{aligned}
\Gamma_{1-p h o n}\left(\xi_{\mathbf{k}}^{(+)}\right)= & 2 Z t^{2} e^{-2 S_{T}} I_{1}(z) \sinh \left(\frac{\beta \bar{\omega}_{0}}{2}\right) g_{1, l=1}\left(\xi_{\mathbf{k}}^{(+)}\right)+ \\
& g^{2} \omega_{0}^{2} e^{4 \alpha}(1-f)^{2} g_{2}\left(\xi_{\mathbf{k}}^{(+)}\right)
\end{aligned}
$$

$\Gamma_{\text {multi-phon }}$ represents the scattering rate by multiphonon processes

$$
\Gamma_{\text {multi-phon }}\left(\xi_{\mathbf{k}}^{(+)}\right)=2 Z t^{2} e^{-2 S_{T}} \sum_{l=2}^{+\infty} I_{l}(z) \sinh \left(\frac{\beta \bar{\omega}_{0} l}{2}\right) g_{1, l}\left(\xi_{\mathbf{k}}^{(+)}\right)
$$

and $\Gamma_{\text {res }}$ denotes a residue term due to the difference between the coherent and the incoherent contribution $^{47}$

$$
\Gamma_{r e s}\left(\xi_{\mathbf{k}}^{(+)}\right)=Z t^{2} e^{-2 S_{T}}\left[I_{0}(z)-1\right] B\left(\xi_{\mathbf{k}}^{(+)}\right)
$$

In the previous equations the function $g_{1, l}\left(\xi_{\mathbf{k}}^{(+)}\right)$reads

$$
\begin{aligned}
g_{1, l}\left(\xi_{\mathbf{k}}^{(+)}\right) & =\left[N_{0}\left(l \bar{\omega}_{0}\right)+n_{F}\left(\xi_{\mathbf{k}}^{(+)}+l \bar{\omega}_{0}\right)\right] B\left(\xi_{\mathbf{k}}^{(+)}+l \bar{\omega}_{0}\right) \\
& +\left[N_{0}\left(l \bar{\omega}_{0}\right)+1-n_{F}\left(\xi_{\mathbf{k}}^{(+)}-l \bar{\omega}_{0}\right)\right] B\left(\xi_{\mathbf{k}}^{(+)}-l \bar{\omega}_{0}\right)
\end{aligned}
$$

and $g_{2}\left(\xi_{\mathbf{k}}^{(+)}\right)$

$$
g_{2}\left(\xi_{\mathbf{k}}^{(+)}\right)=\left[N_{0}+n_{F}\left(\xi_{\mathbf{k}}^{(+)}+\bar{\omega}_{0}\right)\right] B\left(\xi_{\mathbf{k}}^{(+)}+\bar{\omega}_{0}\right)+\left[N_{0}+1-n_{F}\left(\xi_{\mathbf{k}}^{(+)}-\bar{\omega}_{0}\right)\right] B\left(\xi_{\mathbf{k}}^{(+)}-\bar{\omega}_{0}\right) .
$$


Moreover the function $B(x)$ is equal to $2 \pi K(x)$, with $K(x)$ given in eq.(27). The decomposition of the scattering rate in three distinct terms has been introduced in order to simplify the analysis of the results.

We define the scattering rate for the quasi-particles of the lower band

$$
\Gamma_{-}(\mathbf{k})=\tilde{\Gamma}\left(\omega=\xi_{\mathbf{k}}^{(-)}\right)=-2 \Im \Sigma_{r e t}^{(2)}\left(\omega=\xi_{\mathbf{k}}^{(-)}\right)
$$

that turns out to be equal to $\Gamma_{+}(\mathbf{k})$. Thus we can take into account only one scattering rate

$$
\Gamma(\mathbf{k})=\Gamma_{+}(\mathbf{k})=\Gamma_{-}(\mathbf{k})
$$

In Fig. 4 a we report the scattering rate $\Gamma$ for different $e l-p h$ couplings at low temperature. In the normal phase $(\lambda=0.15)$ the scattering rate is zero within $\omega_{0}$ of the chemical potential $\mu$. Therefore at this temperature the main mechanism of energy loss is the spontaneous emission of phonons with frequency $\omega_{0}$ by the quasi-particles (one phonon processes are prevalent). Thus the behavior of the scattering rate is determined by of the Fermi statistics since the quasi-particle excitations within $\omega_{0}$ of the Fermi energy cannot lose energy. ${ }^{46,47}$ In the $C O$ phase $(\lambda=0.50)$ the gap in the scattering rate opens at energies given by the sum of the gap energy (indicated by the arrows in the figure) and the phonon frequency. Since there are not available states within the gap, only the quasi-particles with such energies can be scattered by the residue $e l-p h$ interaction.

In Fig.4b we concentrate on the scattering rate at a fixed $e l-p h$ coupling $(\lambda=0.4)$ for different temperatures. At low $T(T=0.01 W)$ there is the gap due to the Peierls dimerization and the processes of phonon spontaneous emission by the quasi-particles. With rising temperature, we can follow the increase of the quantity $\Gamma$ in the normal phase because of the absorption and emission of phonons. In the normal phase at finite temperatures, these scattering processes are effective also in the energy range around the chemical potential where there are able to enhance the rate. Actually, at higher temperatures $(T=0.14 W)$, the rate is weakly dependent on the energies involved in the phonon scattering. It is confirmed that in this regime of temperatures the single-phonon emission and absorption give an important contribution to the quantity $\Gamma$. 
For large $e l-p h$ couplings and for small polaron excitations, the quantity $\Gamma$ decreases when $T$ increases but it is always larger than $t_{e f f}$, therefore the electronic states lose their individual characteristics and the electron motion is predominantly a diffusive process. A high-temperature expansion ${ }^{37,38,46}$ provides the scattering rate

$$
\Gamma=Z t^{2} \sqrt{\frac{\pi \beta}{\bar{z}}} e^{-\beta \bar{z} / 4}=Z t^{2} \sqrt{\frac{\pi \beta}{4 E_{a}}} e^{-\beta E_{a}},
$$

with $\bar{z}=2 g^{2} \omega_{0}$ and $E_{a}=\bar{z} / 4$. This value coincides with the well-known rate of the Holstein polaron in the "classical" limit, with $E_{a}$ typical activation energy for hopping. ${ }^{23}$

The introduction of the damping allows to improve the approximations of calculation for the spectral properties. This can be carried out substituting in eq.(21) the new Green's function $\tilde{\mathcal{G}}^{(c)}$

$$
\tilde{\mathcal{G}}^{(c)}\left(\mathbf{k}, i \omega_{n}\right)=u_{\mathbf{k}}^{2} \tilde{\mathcal{G}}^{(+)}\left(\mathbf{k}, i \omega_{n}\right)+v_{\mathbf{k}}^{2} \tilde{\mathcal{G}}^{(-)}\left(\mathbf{k}, i \omega_{n}\right) .
$$

for $\mathcal{G}^{(c)}$. The Green's function $\tilde{\mathcal{G}}^{(\nu)}\left(\mathbf{k}, i \omega_{n}\right)$ is expressed as

$$
\tilde{\mathcal{G}}^{(\nu)}\left(\mathbf{k}, i \omega_{n}\right)=\int_{-\infty}^{+\infty} \frac{d \omega}{2 \pi} \frac{\tilde{A}^{(\nu)}(\mathbf{k}, \omega)}{i \omega_{n}-\omega},
$$

where the spectral function $\tilde{A}^{(\nu)}$ is assumed to be

$$
\tilde{A}^{(\nu)}(\mathbf{k}, \omega)=\frac{\Gamma(\mathbf{k})}{[\Gamma(\mathbf{k})]^{2} / 4+\left(\omega-\xi_{\mathbf{k}}^{(\nu)}\right)^{2}},
$$

with $\nu$ standing for + or - . We can determine a new electron spectral function and density of states $N_{\text {Fluct }}$ that includes fluctuation effects beyond the $Z F A$ approach. These effects are able to change the density of states in the Fermi-liquid-like phase. Indeed in this phase it is interesting to study the behavior of the density as function of the temperature at a fixed value of the coupling constant $\lambda$ (see Fig.5a). While the density obtained within the $Z F A$ approach is similar to the bare one (solid line in figure), now $N_{\text {Fluct }}$ shows an enhancement at the Fermi energy in the low temperature regime $(T=0.07 \mathrm{~W})$ and a depression at the same energy in the high $T$ case $(T=0.20 W)$. Actually at low $T$ the processes of phonon spontaneous emission subtract spectral weight to states of energy larger than $\omega_{0}$ 
compared with the chemical potential inducing an increase at the Fermi energies where the states are not damped. At intermediate temperatures $(T=0.12 W)$, where real phonons are present, the absorption and emission processes are effective also around the chemical potential and oppose this enhancement, so that the density of states at the Fermi energy is nearly unchanged. At higher temperatures these processes dominate causing a reduction of the spectral weight at the chemical potential.

In Fig.5b the density of states $N_{\text {Fluct }}$ is plotted as a function of the coupling constant $\lambda$ showing that it is able to capture the features of the intermediate coupling regime. For these values of parameters, the density within the $Z F A$ approach shows a discontinuous transition from a lightly renormalized function to a strongly modified density with a marked reduction at the chemical potential. Due to the included lattice fluctuations, $N_{\text {Fluct }}$ evolves gradually from the bare density to the characteristic small polaron function showing pseudogap features in agreement with previous studies. ${ }^{29-31}$ Not only in the normal phase but also in the $C O$ state, the quantity $\Gamma$ changes the spectral properties. Actually, in the boundary phase region below the transition temperature of the $Z F A$ approach, a pseudogap opens in the density of states as a precursor effect of a gap at lower temperatures and stronger $e l-p h$ couplings.

Thus the lattice fluctuation effects introduced by means a self-energy insertion turn out to be able to correct the drawbacks of the $Z F A$ approach. Next we will focus on the optical properties analyzing the prominent role of the lattice fluctuations in determining the infrared absorption of the system.

\section{OPTICAL PROPERTIES}

In this section we deal with the optical properties near and within the $C O$ phase. Calculating the real part of the conductivity in a regime of linear response reduces to evaluate the retarded current-current correlation function

$$
\Re \sigma_{\alpha, \beta}(\omega)=-\frac{\Im \Pi_{\alpha, \beta}^{r e t}(\omega)}{\omega} .
$$


The electron motion will be supposed to take place between the equivalent $n n$ sites of the cubic lattice, hence the tensor $\sigma_{\alpha, \beta}$ is assumed to be diagonal with mutually equal elements $\sigma_{\alpha, \alpha}$

Performing the two canonical transformations $(4,5)$ and making the decoupling ${ }^{46}$ of the correlation function in the electron and phonon terms through the introduction of $H_{\text {test }}(10)$, we get in Matsubara frequencies

$$
\Pi_{\alpha, \alpha}\left(i \omega_{n}\right)=e^{2} t^{2}\left(-\frac{1}{L}\right) \sum_{i, \delta} \sum_{i^{\prime}, \delta^{\prime}}\left(\delta \cdot \delta^{\prime}\right) \int_{0}^{\beta} d \tau e^{i \omega_{n} \tau} \Phi\left(i, i^{\prime}, \delta, \delta^{\prime}, \tau\right) \Delta\left(i, i^{\prime}, \delta, \delta^{\prime}, \tau\right),
$$

where the function $\Delta$ denotes the electron correlation function

$$
\Delta\left(i, i^{\prime}, \delta, \delta^{\prime}, \tau\right)=\left\langle T_{\tau} \bar{c}_{i}^{\dagger}(\tau) \bar{c}_{i+\delta \hat{\alpha}}(\tau) c_{i^{\prime}+\delta^{\prime} \hat{\alpha}}^{\dagger} c_{i^{\prime}}\right\rangle_{t}
$$

and the function $\Phi$ the phonon correlation function

$$
\Phi\left(i, i^{\prime}, \delta, \delta^{\prime}, \tau\right)=\left\langle T_{\tau} \bar{X}_{i}^{\dagger}(\tau) \bar{X}_{i+\delta \hat{\alpha}}(\tau) X_{i^{\prime}+\delta^{\prime} \hat{\alpha}}^{\dagger} X_{i^{\prime}}\right\rangle_{t}
$$

To derive the optical properties, the role of the damping $\Gamma$ of the particle motion is fundamental. Since the electron correlation function can be expressed as a function of the Green's functions $\mathcal{G}^{(\nu)}$, the effect of the damping ${ }^{46}$ can enter our calculation substituting $\mathcal{G}^{(\nu)}$ for $\tilde{\mathcal{G}}^{(\nu)}$ given by eq.(43). Furthermore, in order to simplify the analysis of our results, we separate $\Phi$ into two contributions

$$
\begin{aligned}
\Phi\left(i, i^{\prime}, \delta, \delta^{\prime}\right) & =\left[\left\langle X_{i}^{\dagger} X_{i+\delta \hat{\alpha}}\right\rangle_{t}\right]^{2}\left\{\Phi\left(i, i^{\prime}, \delta, \delta^{\prime}\right)-\left[\left\langle X_{i}^{\dagger} X_{i+\delta \hat{\alpha}}\right\rangle_{t}\right]^{2}\right\} \\
& =e^{-2 S_{T}}+\left[\Phi\left(i, i^{\prime}, \delta, \delta^{\prime}\right)-e^{-2 S_{T}}\right] .
\end{aligned}
$$

Considering the two terms of eq.(49), the current-current correlation function can be written as

$$
\Pi_{\alpha, \alpha}\left(i \omega_{n}\right)=\Pi_{\alpha, \alpha}^{(1)}\left(i \omega_{n}\right)+\Pi_{\alpha, \alpha}^{(2)}\left(i \omega_{n}\right)
$$

The first term reads

$$
\Pi_{\alpha, \alpha}^{(1)}\left(i \omega_{n}\right)=4 e^{2} t^{2} e^{-2 S_{T}}\left(\frac{1}{L}\right) \sum_{\mathbf{k} \in N Z} \sin ^{2}\left(k_{\alpha}\right) \sum_{\nu_{1}, \nu_{2}} h^{\left(\nu_{1}, \nu_{2}\right)}(\mathbf{k}) S^{\left(\nu_{1}, \nu_{2}\right)}\left(\mathbf{k}, i \omega_{n}\right),
$$


where we have

$$
\begin{gathered}
h^{(+,+)}(\mathbf{k})=h^{(-,-)}(\mathbf{k})=\left(u_{\mathbf{k}}^{2}-v_{\mathbf{k}}^{2}\right)^{2}, \\
h^{(+,-)}(\mathbf{k})=h^{(-,+)}(\mathbf{k})=4 u_{\mathbf{k}}^{2} v_{\mathbf{k}}^{2}
\end{gathered}
$$

with $S^{\left(\nu_{1}, \nu_{2}\right)}\left(\mathbf{k}, i \omega_{n}\right)$ given by

$$
S^{\left(\nu_{1}, \nu_{2}\right)}\left(\mathbf{k}, i \omega_{n}\right)=\int_{0}^{\beta} d \tau e^{i \omega_{n} \tau} \tilde{\mathcal{G}}^{\left(\nu_{1}\right)}(\mathbf{k},-\tau) \tilde{\mathcal{G}}^{\left(\nu_{2}\right)}(\mathbf{k}, \tau) .
$$

The second term of the current-current correlation function is obtained retaining only the main autocorrelation term $i=i^{\prime}$ and $\delta=\delta^{\prime}$

$$
\Pi_{\alpha, \alpha}^{(2)}\left(i \omega_{n}\right)=\left(\frac{2 e^{2}}{Z}\right)\left(\frac{1}{L^{2}}\right) \sum_{\mathbf{k} \in N Z} \sum_{\mathbf{k}_{1} \in N Z} \sum_{\nu_{1}, \nu_{2}} p^{\left(\nu_{1}, \nu_{2}\right)}\left(\mathbf{k}, \mathbf{k}_{1}\right) T^{\left(\nu_{1}, \nu_{2}\right)}\left(\mathbf{k}, \mathbf{k}_{1}, i \omega_{n}\right)
$$

where we have

$$
\begin{aligned}
& p^{(+,+)}\left(\mathbf{k}, \mathbf{k}_{1}\right)=p^{(-,-)}\left(\mathbf{k}, \mathbf{k}_{1}\right)=\left(u_{\mathbf{k}} u_{\mathbf{k}_{1}}-v_{\mathbf{k}} v_{\mathbf{k}_{1}}\right)^{2}, \\
& p^{(+,-)}\left(\mathbf{k}, \mathbf{k}_{1}\right)=p^{(-,+)}\left(\mathbf{k}, \mathbf{k}_{1}\right)=\left(u_{\mathbf{k}} v_{\mathbf{k}_{1}}+u_{\mathbf{k}_{1}} v_{\mathbf{k}}\right)^{2},
\end{aligned}
$$

the function $T^{\left(\nu_{1}, \nu_{2}\right)}\left(\mathbf{k}, \mathbf{k}_{1}, i \omega_{n}\right)$

$$
T^{\left(\nu_{1}, \nu_{2}\right)}\left(\mathbf{k}, \mathbf{k}_{1}, i \omega_{n}\right)=\int_{0}^{\beta} d \tau e^{i \omega_{n} \tau} \tilde{\mathcal{G}}^{\left(\nu_{1}\right)}(\mathbf{k},-\tau) \tilde{\mathcal{G}}^{\left(\nu_{2}\right)}\left(\mathbf{k}_{1}, \tau\right) f_{1}(\tau)
$$

with $f_{1}(\tau)$ given by eq. (30).

We perform the analytic continuation $i \omega_{n} \rightarrow \omega+i \delta$, and, clearly, the conductivity can be expressed as a sum of two terms ${ }^{46}$

$$
\Re \sigma_{\alpha, \alpha}(\omega)=-\frac{\Im\left[\Pi_{\alpha, \alpha}^{r e t(1)}(\omega)+\Pi_{\alpha, \alpha}^{r e t(2)}(\omega)\right]}{\omega}=\Re \sigma_{\alpha, \alpha}^{(c o h)}(\omega)+\Re \sigma_{\alpha, \alpha}^{(i n c o h)}(\omega) .
$$

As in the spectral properties, the appearance of two physically distinct contributions, the coherent and incoherent one, occurs. Actually the first term $\Re \sigma_{\alpha, \alpha}^{(c o h)}$ is due to the charge transfer affected by the interactions with the lattice but not accompanied by processes 
changing the number of phonons. On the other hand, the incoherent term $\Re \sigma_{\alpha, \alpha}^{(i n c o h)}$ in eq. (59) derives from inelastic scattering processes of emission and absorption of phonons. The coherent conductivity is derived as $\Re \sigma_{\alpha, \alpha}^{(c o h)}(\omega)=\left(\frac{4 e^{2} t^{2}}{\omega}\right) e^{-2 S_{T}} \sum_{\nu_{1}, \nu_{2}} \int_{-\tilde{W}}^{0} d \epsilon\left[n_{F}\left(\xi^{\left(\nu_{1}\right)}-\omega\right)-n_{F}\left(\xi^{\left(\nu_{1}\right)}\right)\right] \tilde{C}^{\left(\nu_{1}, \nu_{2}\right)}(\epsilon, \omega) h(\epsilon) A^{\left(\nu_{1}, \nu_{2}\right)}(\epsilon)$,

where $\tilde{C}^{\left(\nu_{1}, \nu_{2}\right)}(\epsilon, \omega)$ is

$$
\tilde{C}^{\left(\nu_{1}, \nu_{2}\right)}(\epsilon, \omega)=\frac{\Gamma(\epsilon)}{\Gamma^{2}(\epsilon)+\left(\xi^{\left(\nu_{2}\right)}-\xi^{\left(\nu_{1}\right)}+\omega\right)^{2}}
$$

and $h(\epsilon)$ is defined as

$$
h(\epsilon)=\left(\frac{1}{N}\right) \sum_{\mathbf{k}} \sin ^{2}\left(k_{\alpha}\right) \delta\left(\epsilon-\tilde{\epsilon}_{\mathbf{k}}\right)
$$

In eq.(60) the function $A^{\left(\nu_{1}, \nu_{2}\right)}(\epsilon)$ is expressed by

$$
A^{(+,+)}(\epsilon)=A^{(-,-)}(\epsilon)=\frac{\epsilon^{2}}{\epsilon^{2}+E^{2}}
$$

and

$$
A^{(+,-)}(\epsilon)=A^{(-,+)}(\epsilon)=\frac{E^{2}}{\epsilon^{2}+E^{2}}
$$

The latter term of the conductivity becomes

$$
\begin{aligned}
\Re \sigma_{\alpha, \alpha}^{(i n c o h)}(\omega)= & \left(\frac{2 e^{2} t^{2}}{\omega}\right) e^{-2 S_{T}} \sum_{\nu_{1}, \nu_{2}} \int_{-\tilde{W}}^{0} d \epsilon \int_{-\tilde{W}}^{0} d \epsilon_{1} g(\epsilon) g\left(\epsilon_{1}\right) R^{\left(\nu_{1}, \nu_{2}\right)}\left(\epsilon, \epsilon_{1}, \omega\right)+ \\
& \left(\frac{2 e^{2} t^{2}}{\omega}\right) e^{-2 S_{T}}\left[I_{0}(z)-1\right] \sum_{\nu_{1}, \nu_{2}} \times \\
& \times \int_{-\tilde{W}}^{0} d \epsilon \int_{-\tilde{W}}^{0} d \epsilon_{1} g(\epsilon) g\left(\epsilon_{1}\right)\left[n_{F}\left(\xi_{1}^{\left(\nu_{2}\right)}-\omega\right)-n_{F}\left(\xi_{1}^{\left(\nu_{2}\right)}\right)\right] C^{\left(\nu_{1}, \nu_{2}\right)}\left(\epsilon, \epsilon_{1}, \omega\right),
\end{aligned}
$$

where $g(\epsilon)$ is the density of states (17), the function $R^{\left(\nu_{1}, \nu_{2}\right)}\left(\epsilon, \epsilon_{1}, \omega\right)$ is given by

$$
R^{\left(\nu_{1}, \nu_{2}\right)}\left(\epsilon, \epsilon_{1}, \omega\right)=2 \sum_{l=1}^{+\infty} I_{l}(z) \sinh \left(\frac{\beta \bar{\omega}_{0} l}{2}\right)\left[J_{l}^{\left(\nu_{1}, \nu_{2}\right)}\left(\epsilon, \epsilon_{1}, \omega\right)+H_{l}^{\left(\nu_{1}, \nu_{2}\right)}\left(\epsilon, \epsilon_{1}, \omega\right)\right]
$$

$C^{\left(\nu_{1}, \nu_{2}\right)}\left(\epsilon, \epsilon_{1}, x\right)$ is 


$$
C^{\left(\nu_{1}, \nu_{2}\right)}\left(\epsilon, \epsilon_{1}, x\right)=\frac{1}{2} \frac{\left[\Gamma(\epsilon)+\Gamma\left(\epsilon_{1}\right)\right]}{\left[\Gamma(\epsilon)+\Gamma\left(\epsilon_{1}\right)\right]^{2} / 4+\left(\xi^{\left(\nu_{1}\right)}-\xi_{1}^{\left(\nu_{2}\right)}+x\right)^{2}}
$$

and

$$
\xi_{i}^{\left(\nu_{j}\right)}=\nu_{j} \sqrt{\epsilon_{i}^{2}+E^{2}} .
$$

We notice that the functions $J_{l}^{\left(\nu_{1}, \nu_{2}\right)}\left(\epsilon, \epsilon_{1}, \omega\right)$

$$
\begin{aligned}
J_{l}^{\left(\nu_{1}, \nu_{2}\right)}\left(\epsilon, \epsilon_{1}, \omega\right)= & C^{\left(\nu_{1}, \nu_{2}\right)}\left(\epsilon, \epsilon_{1}, \omega+l \bar{\omega}_{0}\right)\left[n_{F}\left(\xi_{1}^{\left(\nu_{2}\right)}-l \bar{\omega}_{0}-\omega\right)-n_{F}\left(\xi_{1}^{\left(\nu_{2}\right)}-l \bar{\omega}_{0}\right)\right] \times \\
& \times\left[N_{0}\left(l \bar{\omega}_{0}\right)+n_{F}\left(\xi_{1}^{\left(\nu_{2}\right)}\right)\right]
\end{aligned}
$$

and $H_{l}^{\left(\nu_{1}, \nu_{2}\right)}\left(\epsilon, \epsilon_{1}, \omega\right)$

$$
\begin{aligned}
H_{l}^{\left(\nu_{1}, \nu_{2}\right)}\left(\epsilon, \epsilon_{1}, \omega\right)= & C^{\left(\nu_{1}, \nu_{2}\right)}\left(\epsilon, \epsilon_{1}, \omega-l \bar{\omega}_{0}\right)\left[n_{F}\left(\xi_{1}^{\left(\nu_{2}\right)}+l \bar{\omega}_{0}-\omega\right)-n_{F}\left(\xi_{1}^{\left(\nu_{2}\right)}+l \bar{\omega}_{0}\right)\right] \times \\
& \times\left[N_{0}\left(l \bar{\omega}_{0}\right)+1-n_{F}\left(\xi_{1}^{\left(\nu_{2}\right)}\right)\right]
\end{aligned}
$$

describe phonon absorption and emission processes, respectively.

In the limit of high temperatures and for small polaron excitations, the phononic incoherent absorption is prevalent and in this limit an analytic expansion can be performed. ${ }^{46}$

The internal consistency of our approach is verified by using the sum rule

$$
\int_{0}^{\infty} d \omega \Re \sigma_{\alpha, \alpha}(\omega)=-\frac{\pi}{2} e^{2}<\hat{T}_{\alpha, \alpha}>
$$

where $\left\langle\hat{T}_{\alpha, \alpha}\right\rangle$ is the mean value of component of the kinetic energy equal to one third of eq.(18). Employing the calculated spectra, we have checked tha the two sides of eq.(71) differ in a few per cent.

In Fig. 6 we report the conductivity spectra for $T=0.023 W$ at $e l-p h$ couplings. With rising the $e l-p h$ coupling, a tranfer of spectral weight from low to high energies takes place. The Drude term makes smaller and, in the weak to intermediate $C O$ regime, the optical response is characterized by two components: the remnant Drude-like term and the incipient absorption band centered around the gap energy. For stronger $e l-p h$ couplings, the band is peaked around the gap but it is accompained by a subgap tail due to the lattice 
fluctuations. ${ }^{19,20,48}$ Thus, by increasing the parameter $\lambda$ and by crossing the phase transition, we do not have any sharp changes in the optical spectra but a rather continuous evolution. We point out that in the mean-field approach the infrared absorption occurs only at energies above the gap and it is characterized by the inverse square-root singularity. ${ }^{49}$

In the inset of Fig. 6 the gradual disappearance of the Drude term and the rise of the interband absorption can be followed in a narrow range of energies. At higher temperatures a similar evolution of the bands of the infrared absorption is found by increasing the $e l-p h$ strength. Actually, in both the ranges of temperature, the response in the limit $\omega \rightarrow 0$ is completely suppressed only for strong $e l-p h$ couplings where a well-defined optical gap opens. Furthermore in the $C O$ phase there is a lowering of the height of the interband terms that spread over a wide range of energies.

It is interesting to analyze the conductivity spectra at a fixed $e l-p h$ coupling for different temperatures. We take into account two different regimes: $\lambda=0.4(A \rightarrow C O$ phase $)$ and $\lambda=0.8(B \rightarrow C O$ phase). In the first case (Fig.7), by starting from the normal phase and by lowering the temperature, there is a redistribution of the spectral weight among the two components accompanied by a progressive narrowing of the Drude term. The maximum of the second band slightly shifts towards higher frequencies and the optical gap fully develops at low temperatures well within the $C O$ phase. We stress that these results of the optical absorption are consistent with experimental measurements in dichalcogenides. ${ }^{18}$

In the other regime $(\lambda=0.8)$ very different features of the optical response are obtained (Fig.8). Here the evolution from the $S P$ to $C O$ absorption band is characterized by near peak energy. In the $C O$ phase, below $T_{C O}$, there is a residue of optical response in the limit $\omega \rightarrow 0$ and the optical gap starts to appear for lower temperatures in corrispondence of energies that can decrease with lowering $T$. These results can be closely connected with the conductivity spectra measured in nickelates ${ }^{12,15}$ that reveal the relevance of the lattice degrees in stabilizing the $C O$ phase. 


\section{SUMMARY AND CONCLUSIONS}

We have discussed the phase diagram, the spectral and optical properties of the halffilled spinless Holstein model in three dimensions as a function of the temperature and the $e l-p h$ coupling. The lattice fluctuation effects play a crucial role in determining the density of states and the optical response of the system.

The phase diagram is consistent with previous DMFT results pointing out a strong dependence of the transition temperature on the adiabaticity particularly in the weak to intermediate coupling regime. The density of states captures the features of the intermediate coupling regime in agreement with DMFT studies.

Concerning the optical absorption, we have observed that, with decreasing $T$ or increasing the $e l-p h$ coupling, the ordered phase affects the conductivity spectra inducing a transfer of spectral weight from low to high energies with the opening of an optical gap. The inclusion of

effects of quantum lattice fluctuations is able to smooth the inverse square-root singularity of the mean-field approach and induces a subgap tail absorption. In the weak to intermediate coupling regime, a two-peak structure is obtained within the $C O$ phase at low frequency: the remnant Drude-like term and the incipient absorption band centered around the gap energy. The results obtained in the intermediate and in the strong coupling regime are consistent with experimental conductivity spectra. ${ }^{12,15,18,20}$

In this paper lattice fluctuation effects beyond the variational $Z F A$ approach are included by means of self-energy insertions. At the lowest order the main contribution to the selfenergy is independent on the momentum, however at the same order other terms could be easily included in order to give a non local quantity. Furthermore, the scattering rate can be calculated not only perturbatively but also employing a self-consistent procedure. ${ }^{36-38,46}$ If also the real part of the self-energy is introduced by using the Kramers-Kronig relation, the procedure of calculation could become more complete. The agreement of the results obtained in this paper with other approaches seems to suggest that a second-order perturbation theory on the good $Z F A$ solution can capture the physics of the model in the different regimes of 
the normal and ordered state.

Finally we note that our approach is valid in the infrared range of frequencies where the interband absorption takes place. Thus it is not able to reveal the structures attribuited to collective excitation modes arising from the $C D W$ condensate. . $^{16,17,50}$

\section{FIGURE CAPTIONS}

F1 The phase diagram (solid line) corresponding to $W=6 \omega_{0} . C O$ characterizes the charge ordering phase, the $A$ phase represents the Fermi-liquid-like normal state, the $B$ phase the disordered small polaron normal state (the crossover between the $A$ and $B$ phase is marked by the dashed line). In the inset, the ratio of $T=0$ gap $\Delta$ to the ordering temperature $T_{C O}$ as a function of $\lambda$.

F2 (a) The transition temperature as a function of $\lambda$ for two different values of the adiabaticity ratio: $W=6 \omega_{0}$ (solid line) and $W=3 \omega_{0}$ (dashed line). (b) The variation $\Delta T_{C O}=T_{C O}^{*}-T_{C O}$ of the transition temperatures due to different masses of the oscillator as a function of $\lambda$.

F3 (a) The renormalized density of states at $W=6 \omega_{0}$ and $T=0.023 W$ as a function of the energy (in units of $\omega_{0}$ ) for different $e l-p h$ couplings: $\lambda=0.5$ (solid line), $\lambda=0.35$ (dashed line) and $\lambda=0.15$ (dotted line).

(b)The renormalized density of states at $W=6 \omega_{0}$ and $T=0.14 W$ as a function of the energy (in units of $\omega_{0}$ ) for different $e l-p h$ couplings: $\lambda=0.65$ (solid line), $\lambda=0.6$ (dashed line), $\lambda=0.56$ (dotted line) and $\lambda=0.4$ (dash-dotted line).

F4 (a) The scattering rate at $W=6 \omega_{0}$ and $T=0.023 W$ as a function of the energy (in units of $\left.\omega_{0}\right)$ for different $e l-p h$ couplings: $\lambda=0.5$ (solid line) and $\lambda=0.15$ (dashed line).

(b) The scattering rate at $W=6 \omega_{0}$ and $\lambda=0.4 W$ as a function of the energy (in units of $\left.\omega_{0}\right)$ for different temperatures : $T=0.01 W$ (solid line), $T=0.05 W$ (dashed 
line), and $T=0.14 W$ (dotted line).

The arrows indicate the gap energy.

F5 (a) The new renormalized density of states at $W=6 \omega_{0}$ and $\lambda=0.2$ as a function of the energy (in units of $\omega_{0}$ ) for different temperatures.

(b) The new renormalized density of states at $W=6 \omega_{0}$ and $T=0.2 W$ as a function of the energy (in units of $\omega_{0}$ ) for different $e l-p h$ couplings.

F6 The conductivity up to $6 \omega_{0}$ at different $e l-p h$ couplings. In the inset the conductivity up to $2 \omega_{0}$ at different $e l-p h$ couplings. The conductivities are expressed in units of $\frac{e^{2} \rho}{m t}$, with $m=\frac{1}{2 t}$.

F7 The conductivity (in units of $\frac{e^{2} \rho}{m t}$, with $m=\frac{1}{2 t}$ ) up to $2 \omega_{0}$ at different $e l-p h$ couplings.

F8 The conductivity (in units of $\frac{e^{2} \rho}{m t}$, with $m=\frac{1}{2 t}$ ) up to $16 \omega_{0}$ at different $e l-p h$ couplings.

${ }^{1}$ G. Grüner, in Density Waves in Solids (Addison-Wesley, Reading, MA, 1994).

${ }^{2}$ Charge Density Waves in Solids, edited by Gy. Hutiray and J. Sólyom (Springer-Verlag, Heidelberg, 1985).

${ }^{3}$ One-Dimensional Conductors, by S. Kagoshima, H. Nagasawa, and T. Sambongi (SpringerVerlag, New York, 1988).

${ }^{4}$ J.A. Wilson, F.J. Di Salvo, and S. Machajan, Adv. Phys. 24, 117 (1975).

${ }^{5}$ D. McWhan, R. Fleming, D. Moncton, and F.J. Di Salvo , Phys. Rev. Lett. 45, 269 (1980).

${ }^{6}$ G. Traviglini et al., Solid State Commun. 37, 599 (1981).

${ }^{7}$ L. Testardi, Rev. Mod. Phys. 47, 637 (1975). 
${ }^{8}$ J.M. Tranquada, B. Sternlieb, J. Axe, Y. Nakamura, and S. Vahida, Nature (London) 375, 561 (1995).

${ }^{9}$ J.M. Tranquada, D.J. Buttrey, V. Sachan, and J.E. Lorenzo, Phys. Rev. Lett. 73, 1003 (1994).

${ }^{10}$ S.-H. Lee and S.-W. Cheong, Phys. Rev. Lett. 79, 2514 (1997).

${ }^{11}$ S. Mori, C. Chen, and S.-W. Cheong, Nature (London) 392, 473 (1998).

12 T. Katsufuji, T. Tanabe, T. Ishikawa, Y. Fukuda, and Y. Tokura, Phys. Rev. B 54, 14230 (1996).

${ }^{13}$ P. Calvani, G. De Marzi, P. Dore, S. Lupi, P. Maselli, F. D’Amore, S. Gagliardi, and S.-W. Cheong, Phys. Rev. Lett. 81, 4504 (1998).

${ }^{14}$ K.H. Kim, S. Lee, T.W. Noh, and S.-W. Cheong, Phys. Rev. Lett. 88, 167204 (2002).

15 J.H. Jung, D.-W. Kim, T.W. Noh, H.C. Kim, H.-C. Ri, S.J. Levett, M.R. Lees, D. Mck. Paul, and G. Balakrishnan, Phys. Rev. B 64, 165106 (2001).

${ }^{16}$ G. Grüner, Rev. Mod. Phys. 60, 1129 (1988).

${ }^{17}$ L. Degiorgi, B. Alavi, G. Mihály, and G. Grüner, Phys. Rev. B 44, 7808 (1991).

${ }^{18}$ V. Vescoli, L. Degiorgi, H. Berger and L. Forró, Phys. Rev. Lett. 81, 453 (1998).

${ }^{19}$ R.H. McKenzie and J.W. Wilkins, Phys. Rev. Lett. 69, 1085 (1992).

${ }^{20}$ L. Degiorgi, St. Thieme, B. Alavi, G. Grüner, R. H. McKenzie, K. Kim, and F. Levy, Phys. Rev. B 52, 5603 (1995).

${ }^{21}$ A. Schwartz, M. Dressel, B. Alavi, A. Blank, S. Dubois, G. Grüner, B.P. Gorshunov, A.A. Volkov, G.V. Kozlov, S. Thieme, L. Degiorgi, and F. Lévy, Phys. Rev. B 52, 5643 (1995).

${ }^{22}$ P. Calvani, A. Paolone, P. Dore, S. Lupi, P. Maselli, P.G. Medaglia, and S.-W. Cheong, Phys. Rev. B 54, R9592 (1996).

23 T. Holstein, Ann. Phys. (Leipzig) 8, 325 (1959); ibidem 8, 343 (1959). 
${ }^{24}$ F. Marsiglio, Phys. Rev. B 42, 2416 (1990).

${ }^{25}$ R.M. Noack, D.J. Scalapino, and R.T. Scalettar, Phys. Rev. Lett. 66, 778 (1991).

${ }^{26}$ M. Vekić, R.M. Noack, and S.R. White, Phys. Rev. B 46, 271 (1992); M. Vekić and S.R. White, ibid. 48, 7643 (1993).

${ }^{27}$ J.K. Freericks, M. Jarrell, and D.J. Scalapino, Phys. Rev. B 48, 6302 (1993).

${ }^{28}$ P. Niyaz, J.E. Gubernatis, R.T. Scalettar, and C.Y. Fong, Phys. Rev. B 48, 16011 (1993).

${ }^{29}$ S. Ciuchi and F. de Pasquale, Phys. Rev. B 59, 5431 (1999).

${ }^{30}$ S. Blawid and A. Millis, Phys. Rev. B 62, 2424 (2000).

${ }^{31}$ S. Blawid and A. Millis, Phys. Rev. B 63, 115114 (2001).

${ }^{32}$ H. Zheng, D. Feinberg, and M. Avignon, Phys. Rev. B 39, 9405 (1989).

33 J.E. Hirsch and E. Fradkin, Phys. Rev. Lett. 49, 402 (1982); J.E. Hirsch and E. Fradkin, Phys. Rev. B 27, 4302 (1983).

${ }^{34}$ R.H. McKenzie, C.J. Hamer, and D.W. Murray, Phys. Rev. B 53, 9676 (1996); R.J. Bursill, R.H. McKenzie, and C.J. Hamer, Phys. Rev. Lett. 80, 5607 (1998).

${ }^{35}$ A. Weisse and H. Fehske, Phys. Rev. B 58, 13526 (1998).

${ }^{36}$ J. Schnakenberg, Z. Phys. 190, 209 (1966).

${ }^{37}$ J. Loos, Z. Phys. B 92, 377 (1993).

38 J. Loos, Z. Phys. B 96, 149 (1994).

${ }^{39}$ L. P. Kadanoff and G. Baym, Quantum Statistical Mechanics (Reading Massachusetts, Benjamin/Cumming Publishing Company, 1962).

${ }^{40}$ H. Fehske, J. Loss, and G. Wellein, Z. Phys. B 104, 619 (1997).

${ }^{41}$ I. J. Lang and Yu. A. Firsov, Soviet Physics JETP 16, 1301 (1963); Yu. A. Firsov, Polarons 
(Moskow, Nauka, 1975).

${ }^{42}$ E.N. Economou, Green's Functions in Quantum Physics (Springer Verlag, Berlin ,1983).

${ }^{43}$ A. Georges, G. Kotliar, W. Krauth, and M.J. Rozenberg, Rev. Mod. Phys. 68, 13 (1996).

${ }^{44}$ M. Imada, A. Fujimori, and Y. Tokura, Rev. Mod. Phys. 70, 1039 (1998).

${ }^{45}$ C.A. Perroni, V. Cataudella, G. De Filippis, G. Iadonisi, V. Marigliano Ramaglia, and F. Ventriglia, Phys. Rev. B 66, 184409 (2002).

${ }^{46}$ C.A. Perroni, G. De Filippis, V. Cataudella, and G. Iadonisi, Phys. Rev. B 64, 144302 (2001).

${ }^{47}$ G. Mahan, Many-Particle Physics, 2nd ed. (Plenum Press, New York, 1990).

${ }^{48}$ K. Kim, R.H. McKenzie, and J.W. Wilkins, Phys. Rev. Lett. 71, 4015 (1993).

${ }^{49}$ P.A. Lee, T.M. Rice, and P.W. Anderson, Solid State Commun. 14, 703 (1974).

${ }^{50}$ N. Kida and M. Tonouchi, Phys. Rev. B 66, 24401 (2002). 


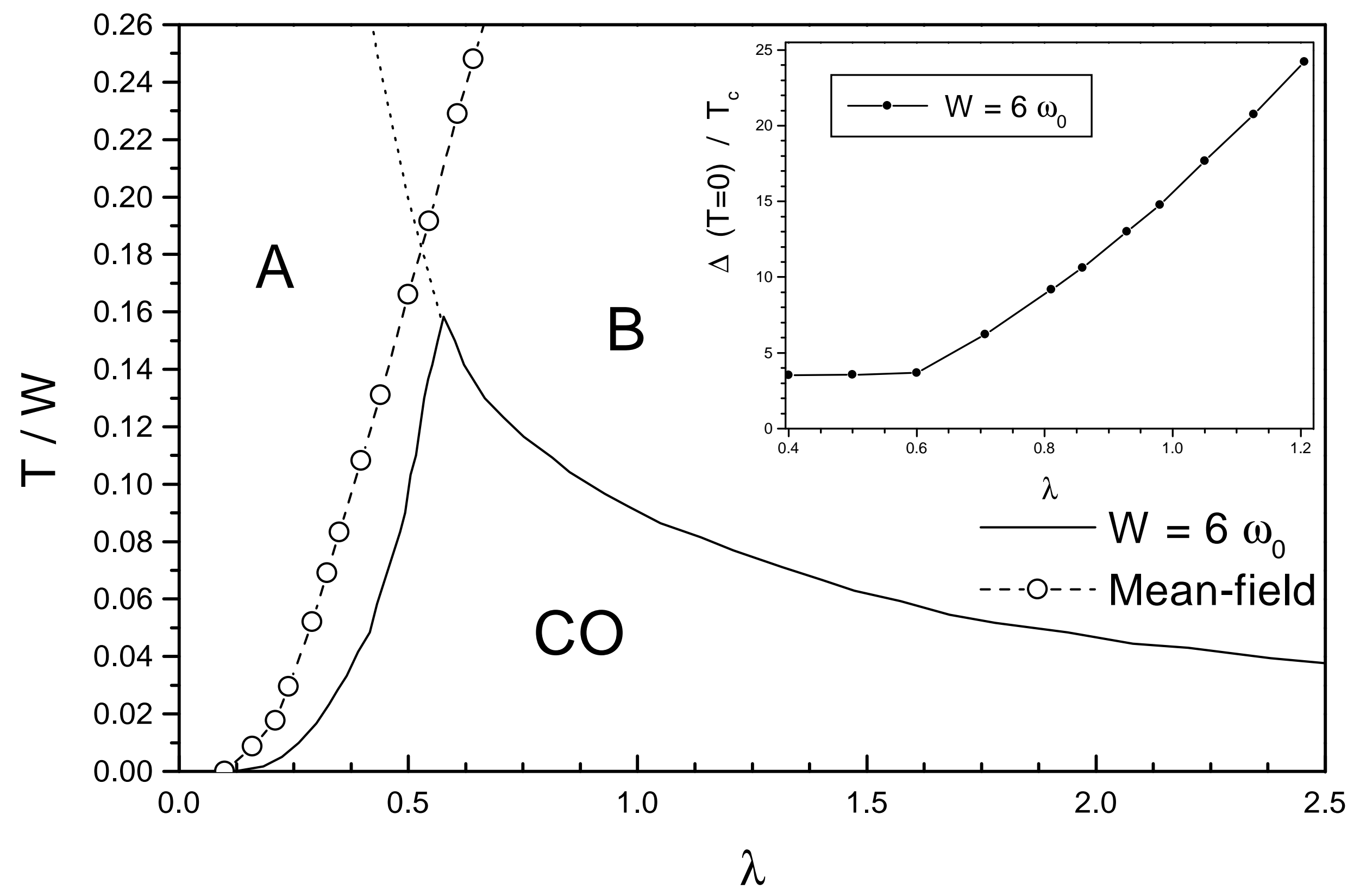



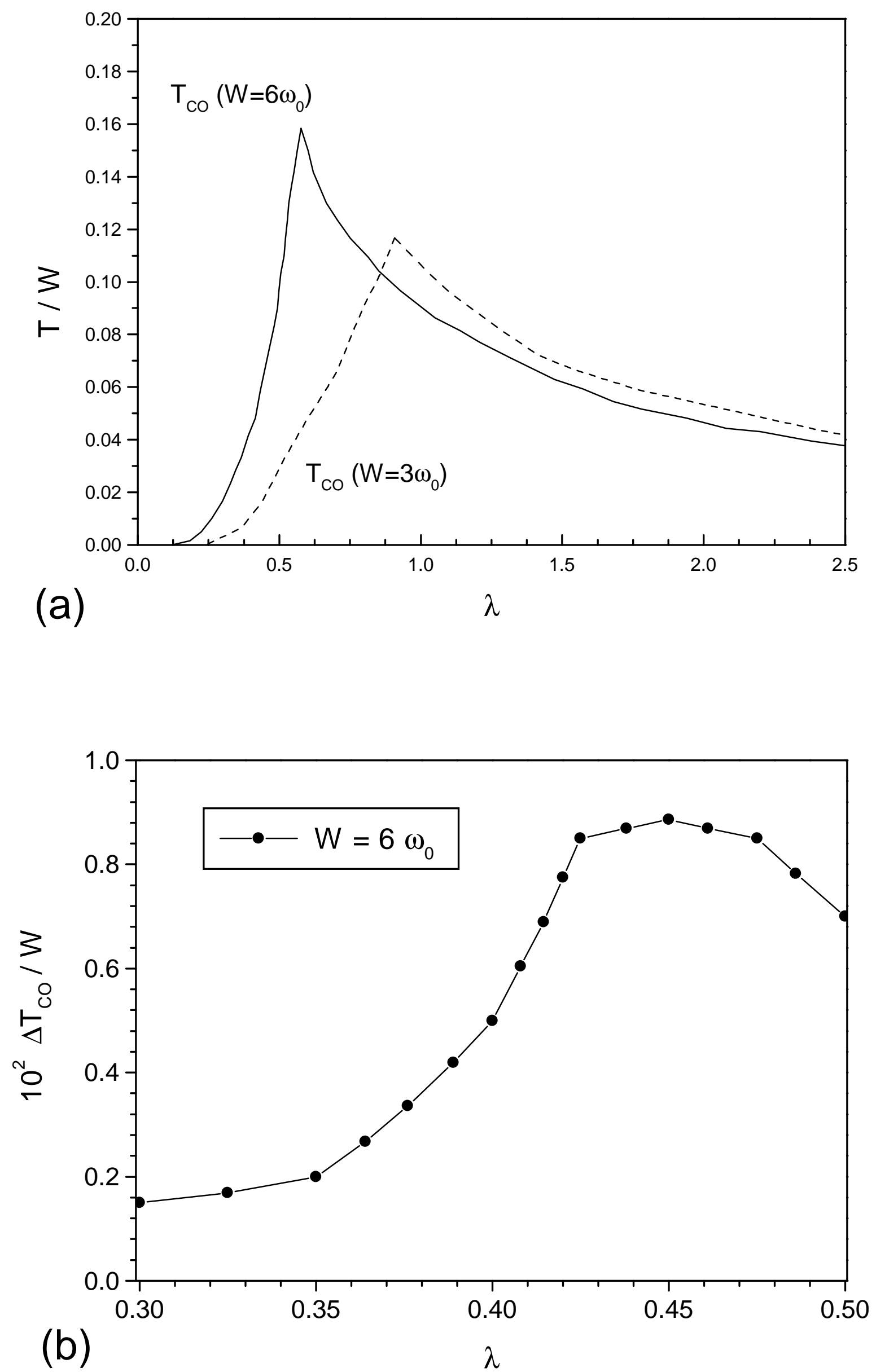

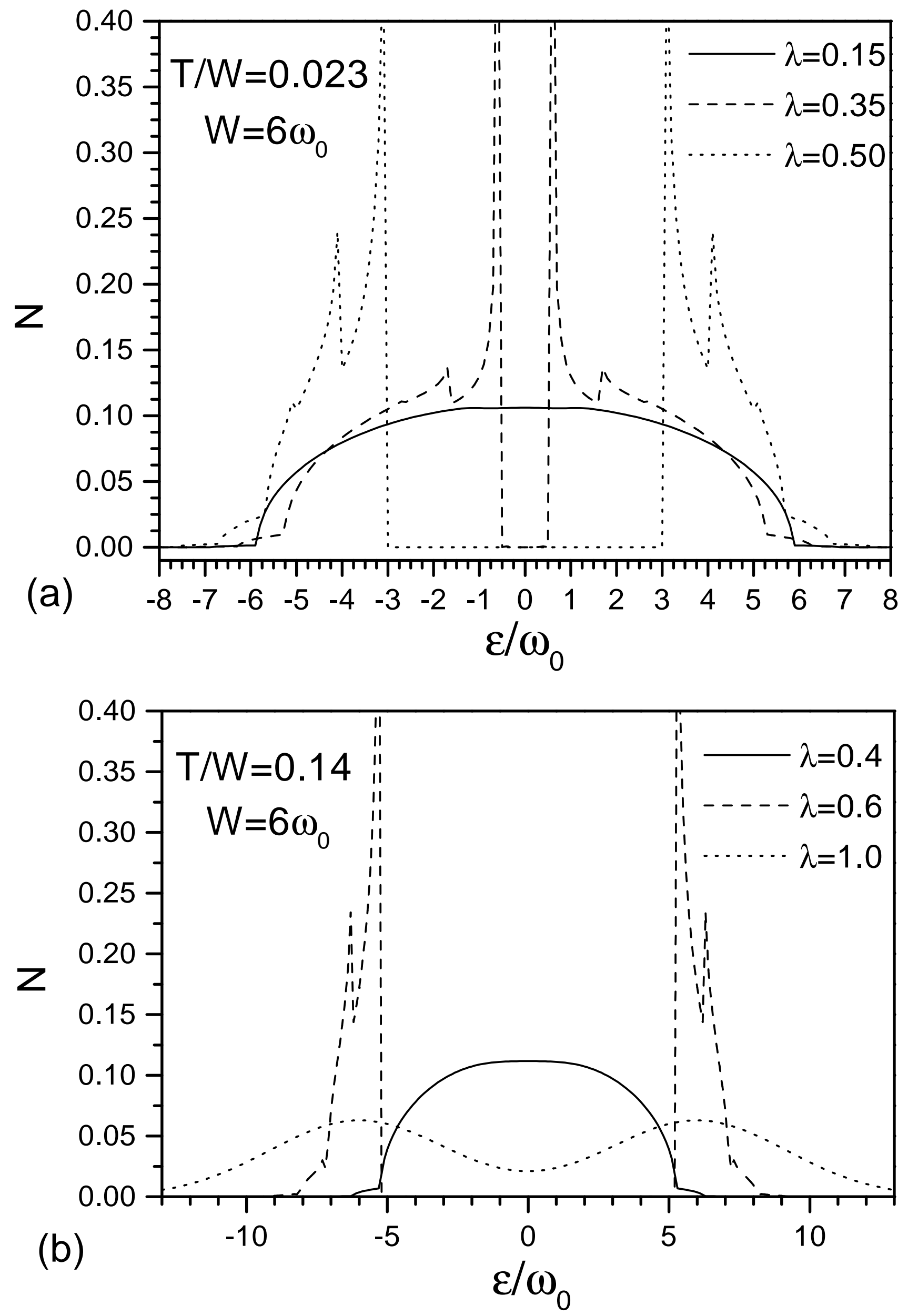

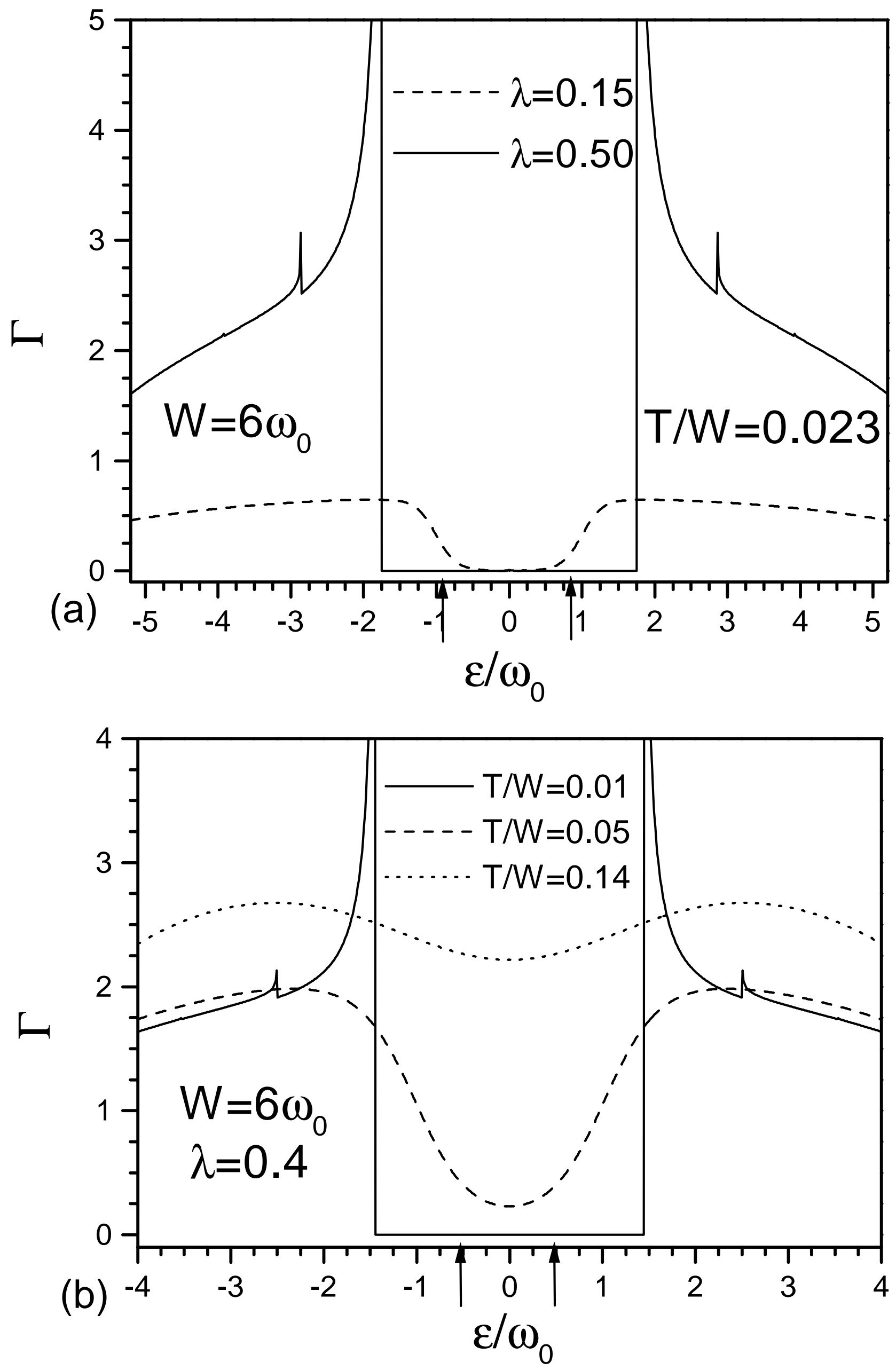

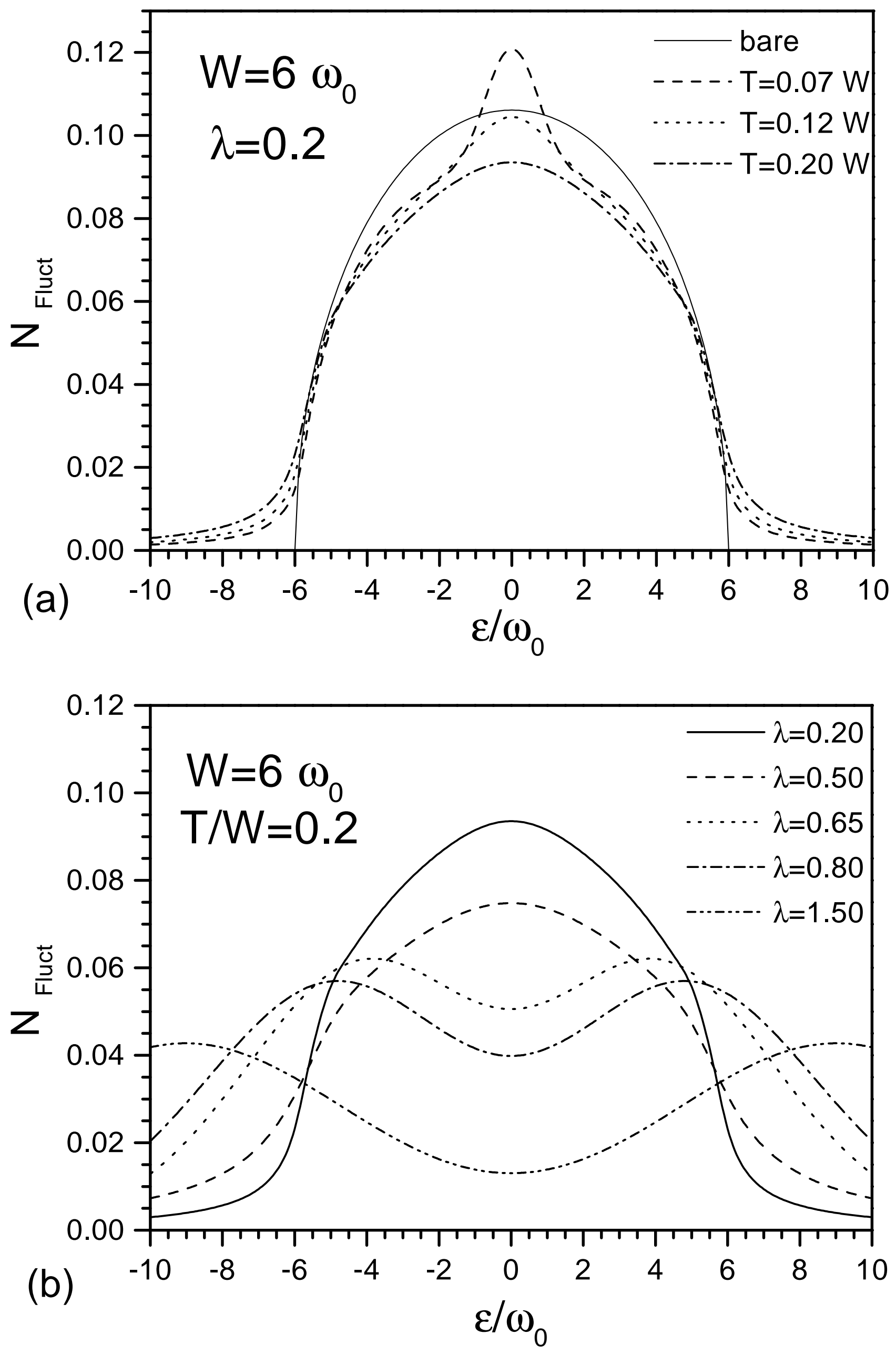


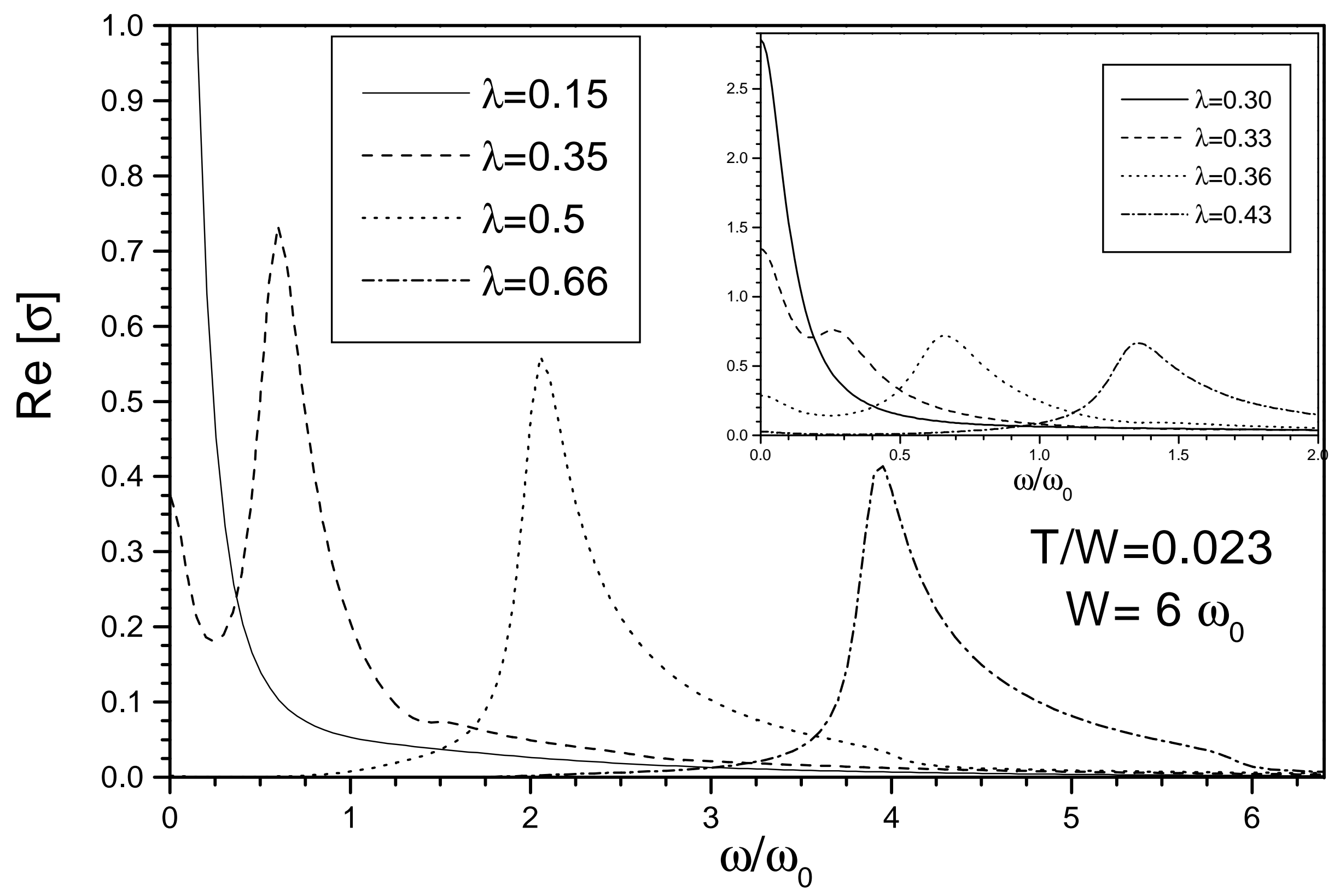




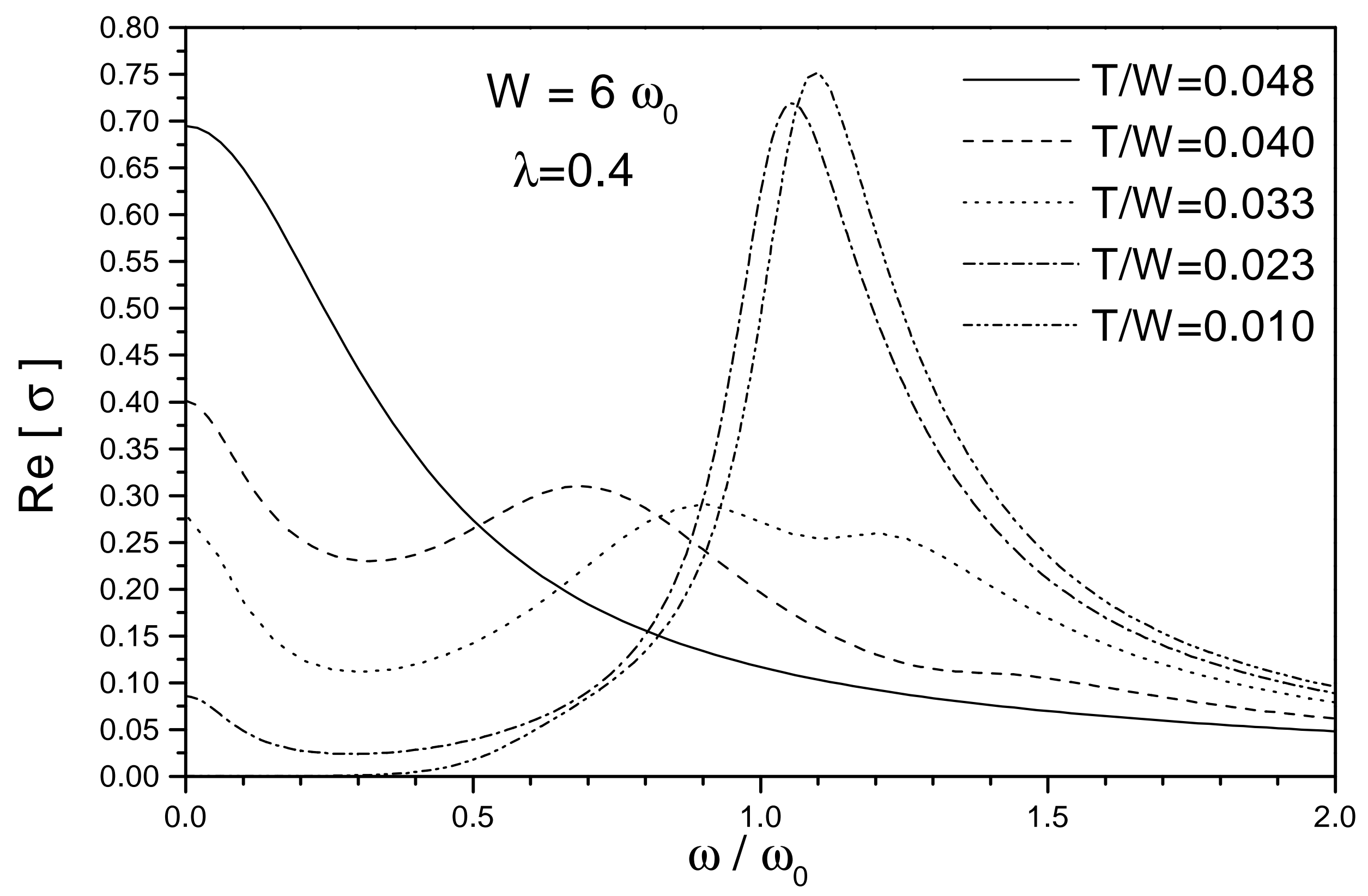




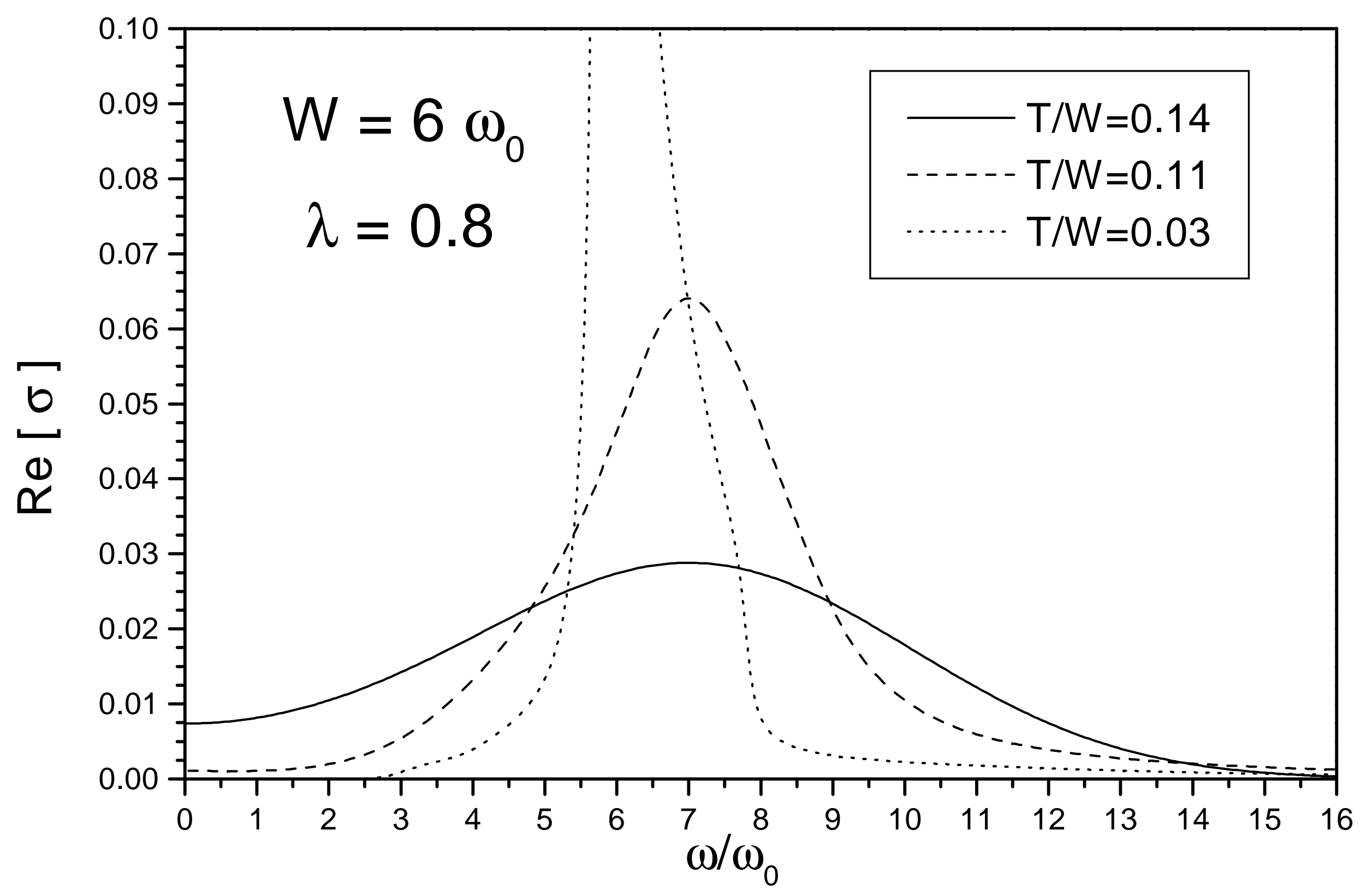

